\title{
Prospecções para desenvolvimento de políticas públicas de formação de profissionais de saúde a partir da análise do cenário brasileiro de competências
}

\section{| ${ }^{1}$ Thaís Branquinho Oliveira Fragelli, ${ }^{2}$ Helena Eri Shimizu |}

Resumo: Quando se refere à Saúde Coletiva, a literatura aponta para a necessidade de profissionais que sejam capazes de atuar em diferentes contextos. Nesta perspectiva, o tema "competência" emerge do contexto administrativo para a saúde, de maneira a promover debates no campo de desenvolvimento de recursos humanos e formação profissional. Este artigo tem por objetivo apresentar um panorama do cenário brasileiro sobre competências profissionais em saúde para melhor entendimento do constructo em nível nacional e propor futuras prospecçōes. $\mathrm{O}$ estudo utilizou dados do Diretório de Grupos de Pesquisa do Conselho Nacional de Pesquisa (CNPq), da base de dados do Departamento de Ciência e Tecnologia do Ministério da Saúde (Sistema Pesquisa em Saúde), do Banco de Teses e de Dissertaçōes da Coordenação de Aperfeiçoamento de Pessoal de Nível Superior (CAPES) e SciELO. Observou-se desigualdade na distribuição de pesquisadores e produção científica pouco incentivada. Indica-se, assim, a necessidade de maiores esforços gerenciais no campo de saúde para a construção do tema.

> Palavras-chave: competências; profissional de saúde; políticas públicas.
1 Mestre em Psicologia Social, do Trabalho e das Organizações (UnB), Doutora em Ciências da Saúde pela Universidade de Brasília. Endereço eletrônico: thaisbranquinho@hotmail.com

2 Doutora em Enfermagem pela Universidade de São Paulo, professor associado da Universidade de Brasília (UnB) Endereço eletrônico: shimizu@ unb.br
Recebido em: 23/01/2011. Aprovado em: 10/12/2012. 


\section{Introdução}

Considerando as transformações que vêm ocorrendo no modelo de atenção à saúde, há uma exigência de novos modos de trabalho respaldados em novos modos de formação e de gestão. Nesta perspectiva, Hennington (2008) afirma que o profissional deve ser capaz de atuar dentro de uma realidade complexa, caminhando para além das prescrições, de maneira a criar, improvisar, construir o curso das ações e definir o melhor modo de trabalhar para atender cada contexto específico (BRASIL, 2004).

Nesta perspectiva, Battel-Kirk et al. (2009) afirmam que uma força de trabalho competente tem o conhecimento e as habilidades necessárias para traduzir a teoria em política, e a pesquisa em ação efetiva, e constitui elemento fundamental para o crescimento e desenvolvimento da promoção da saúde.

Lilley e Stewart (2009), por sua vez, descrevem em seu artigo que o sucesso do sistema de saúde depende de profissionais de saúde qualificados. E Nascimento e Oliveira (2010) complementam que desenvolvimento de competências apresentase como uma nova perspectiva para a formação dos profissionais de saúde, não só por incentivar a reflexão crítica, mas por facilitar a construção de respostas às exigências impostas pelo atual cenário de mudanças sociais.

Diante de tais aspectos, encontram-se na literatura várias definições para a compreensão do termo "competências" (LE DEIST; WINTERTON, 2005; ALAINATI; ALSHAWI; AL-KARAGHOULI, 2010; McMULLAN, 2005; SANDBERG, 2000). Dentre eles, destaca-se a definição de Gonczi (1996), que afirma que competência é o domínio de atributos (conhecimentos, habilidades, valores e atitudes) que se combinam de diversas maneiras para a realização de tarefas em diferentes situações. Assim, segundo o autor, um indivíduo competente seria aquele que possui os atributos necessários para o desempenho do trabalho de acordo com a norma apropriada dentro de um contexto específico.

Beckett (2005) afirma que competência constitui a capacidade de "ler o momento ","um saber como" e "um saber por que", e chama a atenção para uma capacidade reflexiva que antecipa ações. Além disso, discussões têm sido construídas de maneira a relacionar o construto competências e Saúde Coletiva como a Galway Consensus Conference, ocorrida em 2008, evento do qual participaram vários países, com o objetivo de promover, por meio de um intercâmbio global, uma visão compartilhada do termo "competência" e o 
desenvolvimento da capacidade da força de trabalho entre países e continentes (ALLEGRANTE et al., 2009; BARRY et al., 2009).

Diante das consideraçôes expostas, este artigo tem o objetivo de apresentar um panorama do cenário brasileiro sobre competências profissionais em saúde, para melhor entendimento do constructo em nível nacional e propor futuras prospecções. Em seguida, realizou-se um roadmapping, que é um método para desenvolver representaçōes gráficas simplificadas e fornecer um quadro prospectivo. Originase de um neologismo em inglês que inicialmente se relacionava ao planejamento tecnológico, de maneira a identificar, selecionar e desenvolver as alternativas tecnológicas voltadas às necessidades de produção das empresas (SENAI, 2008).

\section{Material e métodos}

Este estudo foi realizado no Diretório de Grupos de Pesquisa do Conselho Nacional de Pesquisa (CNPq), na base de dados do Departamento de Ciência e Tecnologia do Ministério da Saúde (Sistema Pesquisa em Saúde), no Banco de Teses e de Dissertações da Coordenação de Aperfeiçoamento de Pessoal de Nível Superior (CAPES) e na base de dados SciELO (BRASIL, 2010a, 2010b, 2010c; SCIELO, 2010). Foram identificados dados sobre a atividade acadêmica, os investimentos, os atores no processo de produção de conhecimento e a produção científica. Em seguida, os resultados foram analisados com o objetivo de construir cenários futuros e ações necessárias por meio de roadmapping.

Para a pesquisa no Diretório de Grupos de Pesquisa do CNPq, as variáveis analisadas foram: 1) o quantitativo de grupos; e 2) a distribuição regional. $\mathrm{O}$ critério de seleção se condicionou à presença do tema competência em pelo menos uma linha de pesquisa, sem restrição de áreas, com a finalidade de compará-las com a área de Saúde Coletiva.

A pesquisa no Banco de Teses e Dissertações da Capes teve o objetivo de comparar diferentes áreas com o campo de Saúde Coletiva, por isso não se utilizou o filtro "ciências da saúde". Como critério de seleção, os trabalhos deveriam ter como objeto de estudo o tema "competências", sendo considerados aqueles que tivessem o termo em qualquer um dos seguintes campos: título, palavras-chave ou resumo. Não foi estabelecido o limite de data.

O Sistema Pesquisa em Saúde (DECIT/SCTIE/MS), criado em 2002, é um sistema de informaçôes sobre projetos de pesquisas apoiados pelo Departamento 
de Ciência e Tecnologia (DECIT/ SCTIE/ MS) com a colaboração do CNPq, FINEP, UNESCO, OPAS, Fundações de Amparo à Pesquisa, Secretarias Estaduais de Saúde e de Ciência e Tecnologia (BRASIL, 2010b). Aqui a pesquisa se limitou a verificar os projetos financiados e as instituições participantes dos mesmos.

Para os artigos da base do SciELO, foram selecionados a produção a partir de 2000, sendo descartados aqueles que fossem eminentemente teóricos ou de revisão. As palavras-chave utilizadas foram "competência" e "competências profissionais". Os artigos deveriam ter como objeto de estudo o tema "competência", com foco nas áreas da saúde, e o termo deveria estar presente em um dos seguintes campos: título, resumo ou palavras-chave.

Em seguida, será apresentado um resumo dos principais indicadores levantados em cada uma dessas fontes de pesquisa.

\section{Resultados}

\section{Grupos de pesquisa cadastrados no $\mathrm{CNPq}$}

Foram encontrados 12 grupos cadastrados. A distribuição regional se concentrou predominantemente em Minas Gerais, São Paulo, Santa Catarina, Distrito Federal e Rio de Janeiro, como pode ser visualizado no gráfico 1.

\section{Gráfico 1. Distribuição dos grupos de pesquisa por área profissional}

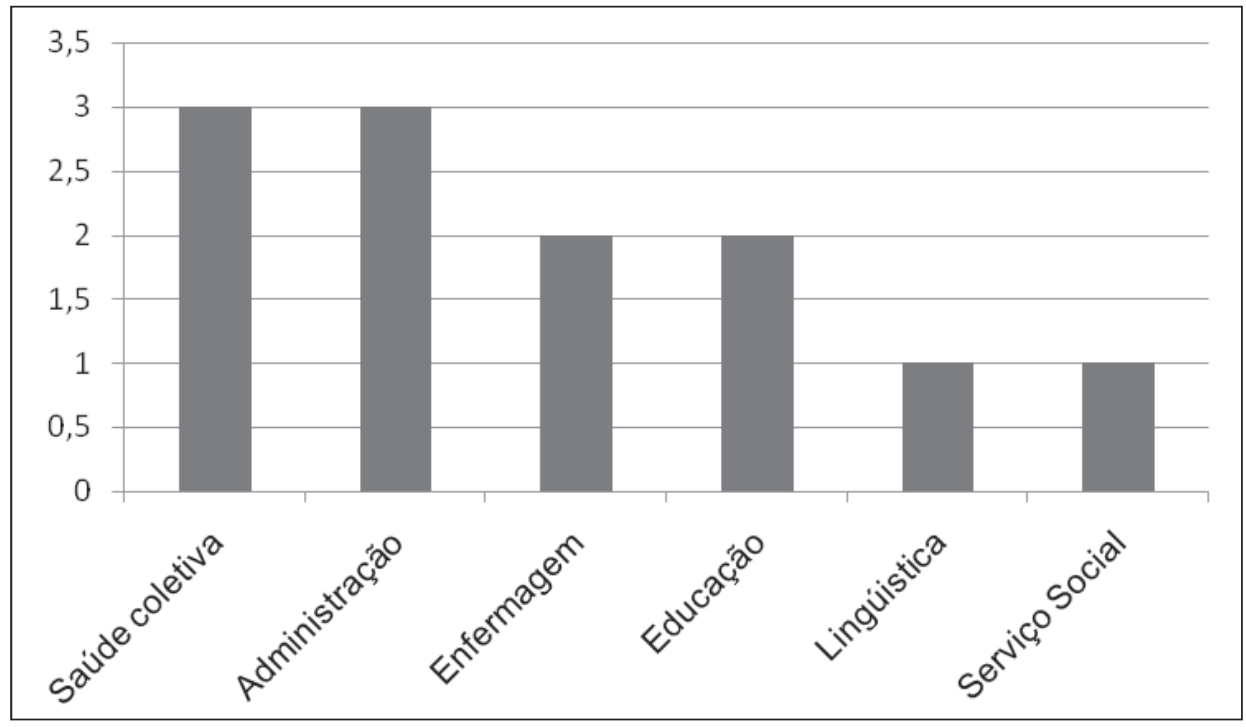


Dos 12 grupos de pesquisa encontrados sobre o tema, apenas três são de Saúde Coletiva e dois de Enfermagem. Na análise do gráfico 1, percebe-se que a Saúde Coletiva tem representatividade semelhante à Administração, mas no gráfico 2 os recursos humanos de pesquisadores e estudantes estão regionalmente mal distribuídos, restringindo-se apenas em alguns centros.

\section{Gráfico 2. Distribuição dos recursos humanos em pesquisa}

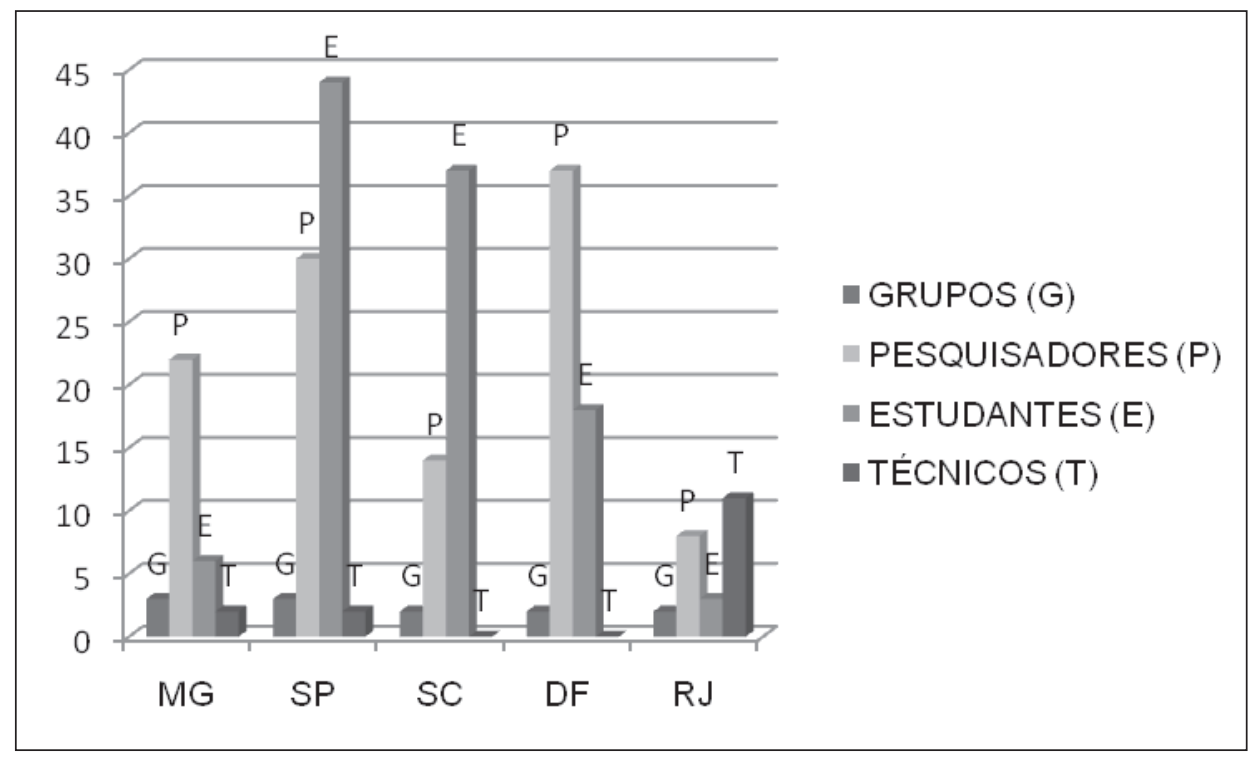

\section{Banco de teses e dissertações da CAPES}

Foram encontrados 80 trabalhos distribuídos em 63 dissertações e 17 teses. Considerando o ano de defesa, observou-se que 14 foram defendidas em 2007, seguido do ano de 2008, com 13, e 2009 com 12. Os anos de 2000, 1997 e 1996 foram os que apresentaram menos defesas, uma cada.

Dentre as instituições que mais apresentaram produção está a USP com 13 trabalhos, sendo sete dissertações e seis teses, conforme mostrado na tabela 1. Observa-se, ainda, predomínio de instituições particulares sobre as públicas. 
Tabela 1. Distribuição de teses e dissertações por instituição

\begin{tabular}{lccc}
\hline INSTITUIÇÃO & MESTRADO & DOUTORADO & PRODUÇÃO \\
\hline USP & 7 & 6 & 13 \\
UNIHORIZONTES & 7 & 0 & 7 \\
PUC/SP & 4 & 1 & 5 \\
UFSC & 5 & 0 & 5 \\
UFRJ & 1 & 3 & 4 \\
ESTÁCIO DE SÁ & 3 & 0 & 3 \\
MACKENZIE & 3 & 0 & 3 \\
UNIFACS & 3 & 0 & 3 \\
UTFPR & 3 & 0 & 3 \\
CEFET/RJ & 2 & 0 & 2 \\
PUC/PR & 2 & 0 & 2 \\
UFPb & 2 & 0 & 2 \\
UFMG & 0 & 2 & 2 \\
UFRS & 1 & 1 & 2 \\
UnB & 1 & 1 & 22 \\
Demais Instituições & 19 & 3 & 80 \\
\hline Total & 63 & 17 & 22 \\
\hline
\end{tabular}

Com relação à distribuição conforme a área de conhecimento, nas Ciências da Saúde, oito trabalhos foram de Enfermagem, dois de Educação Física e um de Odontologia. A Saúde Coletiva apresentou quatro trabalhos, sendo que um de mestrado e três de doutorado. A distribuição completa de todas as áreas é mostrada no gráfico 3 . 


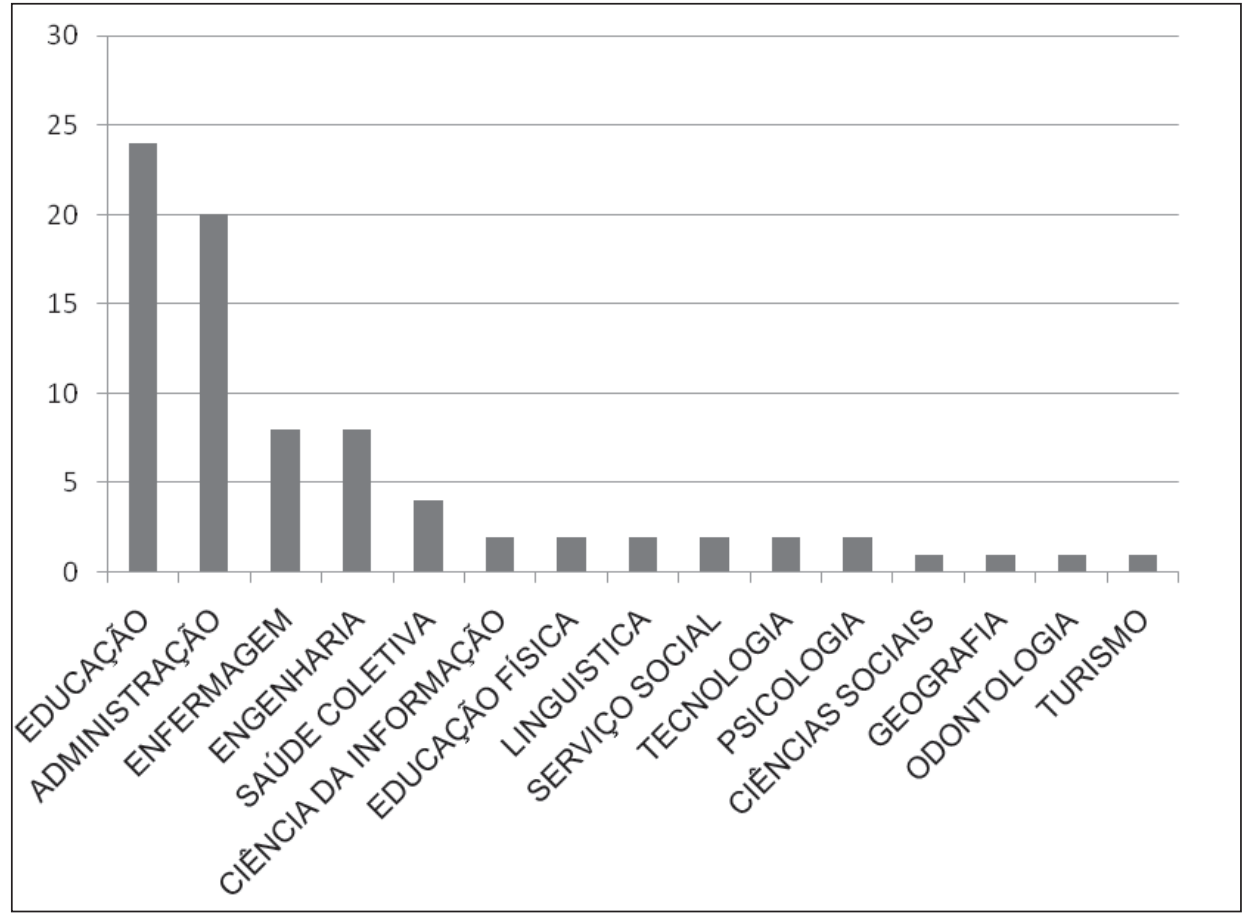

\section{Sistema Pesquisa em Saúde (DECIT/SCTIE/MS)}

Foram encontrados quatro projetos, sendo três do edital PPSUS e um de edital de Fomento Nacional, totalizando R \$ 152.515,38 em recursos disponibilizados. As instituições participantes dos projetos encontrados e suas respectivas unidades federativas foram UNIVALI (SC) em 2003 e 2004; UERN (RN) em 2004; e o Instituto de Saúde (SP) em 2005.

Com relação aos projetos de pesquisa na área de saúde apoiados pelo governo, há apenas quatro editais desde 2002, destacando-se também a má distribuição pelas nas unidades federativas. 


\section{Artigos da base de dados SciELO}

Conforme os critérios de inclusão e exclusão, foram encontrados 15 trabalhos. $\mathrm{O}$ periódico com mais publicaçóes foi a Revista da Escola de Enfermagem da USP, com quatro artigos, seguida da Revista Brasileira de Enfermagem, com três, e as revistas da Escola Anna Nery e Texto e Contexto em Enfermagem, com dois artigos cada. As revistas Saúde e Sociedade, Ciência e Saúde Coletiva, Physis e Interface tiveram cada um artigo.

$\mathrm{Na}$ análise da afiliação dos autores, a USP obteve maior citação, com dez autores; UNIVALI teve cinco; CESGRANRIO, quatro; e UERS, três. UnB, UFAC e UFRJ apresentaram dois cada e Ministério da Saúde, Estácio de Sá, UFMT e IPDC, um cada.

Quanto à titulação dos autores, foram identificados 17 doutores, sete mestres e oito graduados e especialistas. E com relação às áreas de concentração de cada titulação, observou-se que a área de Enfermagem foi mais expressiva, com 16 autores, seguida de Saúde Coletiva, com quatro, e Educação, com dois. As áreas de Filosofia, Psicologia, Engenharia e Administração apareceram com um autor cada. E ainda cinco autores não tinham suas áreas especificadas.

\section{Roadmapping}

Com o objetivo de desenvolver uma representação gráfica que indicasse o cenário descrito anteriormente, optou-se por realizar a técnica de roadmapping. Assim, é apresentado em sua linha superior o objetivo da representação gráfica; na primeira coluna, a situação atual diagnosticada a partir do levantamento mostrado anteriormente; na segunda coluna, sugestões de ações; e na última coluna, um cenário a ser conseguido a partir da efetivação das ações propostas. 
Objetivo: Verificar o cenário para futuras pesquisas sobre competências profissionais em saúde pública e propor sugestões para o fortalecimento do campo científico no desenvolvimento dos recursos humanos em saúde coletiva.

Situação Atual

Poucos grupos

de pesquisa e

desigualmente

localizados

Poucas dissertações

e teses e artigos

produzidos com

concentração em

saúde coletiva

Poucos projetos

financiados no tema
Possíveis Ações

Cenário Futuro

Ampliar os investimentos no tema com parte dos recursos destinados exclusivamente para as regiões Norte e Nordeste.

Induzir pesquisas prioritárias envolvendo a saúde coletiva e o desenvolvimento de recursos humanos

Conscientização dos gestores sobre a necessidade de ampliação do número de projetos relacionados às competências para Saúde Coletiva.
Desenvolvimento de pesquisas em nível nacional.

Mais atores das demais regiões com possibilidade de desenvolvimento de áreas correlatas.

Fortalecimento da área com aumento da produção científica. 


\section{Conclusão}

Considerando a necessidade de profissionais preparados para a nova realidade do modelo assistencial e do contexto epidemiológico, como exposto na introdução do presente artigo, julga-se importante a promoção de estudos e pesquisas na área de desenvolvimento de recursos humanos em saúde.

Para tanto, o tema "competências" tem se mostrado uma preocupação internacional, considerando o movimento da Galway Consensus Conference, realizada em 2008, em que representações de diversos países se reuniram para discutir o atual cenário de saúde e a necessidade de profissionais preparados para lidar com as novas demandas do setor. Assim, o estudo apresentado teve o objetivo de não apenas expor o panorama brasileiro com relação ao tema, mas apontar diretrizes nacionais para futuras prospecções acerca do tema e discussões que favoreçam o desenvolvimento dos recursos humanos em saúde.

Nesta perspectiva, optou-se pela realização da revisão do cenário, conjuntamente com a sistematização dos dados, e posterior proposição visual por meio do roadmapping, técnica segundo a qual é possível visualizar graficamente, de maneira resumida, o que se tem, as estratégias possíveis e os prováveis resultados advindos das ações propostas.

Conclui-se, então, que o tema ainda é pouco explorado nos campos de Saúde Coletiva em nível nacional e desigualmente distribuído pelas unidades federativas, havendo necessidade de mais esforços por parte de gestores, por meio de maior incentivo a pesquisas, com vistas ao desenvolvimento do campo, ainda em construção e, consequentemente, para o fortalecimento do sistema público de saúde. ${ }^{1}$

\section{Referências}

ALAINATI S.; ALSHAWI S.N.; AL-KARAGHOULI W. The effect of education and training on competency. In: EUROPEAN AND MEDITERRANEAN CONFERENCE ON INFORMATION SYSTEMS, 2010, Abu Dhabi, Anais... Abu Dhabi: EMCIS. 9p.

ALLEGRANTE, J.P. et al. Toward International Collaboration on Credentialing in Health Promotion and Health Education: The Galway Consensus Conference. Health Education \& Behavior. New York, n.36, v.3, p. 427-438, 2009.

BARRY, M.M. et al. The Galway Consensus Conference: international collaboration on the development of core competencies for health promotion and health education. Global Health Promotion. Toronto, n.16, v.2, p. 05-11, 2009.

BATTEL-KIRK, B. et al .A review of the international literature on health promotion competencies: identifying frameworks and core competencies. Global Health Promotion. Toronto, n.16, v.2, p.12-20, 2009. 
BECKETT, D. Holistic competence: putting judgements first. Asia Pacific Education Review. Seoul, n. 9, v.1, p.21-30, 2008.

BRASIL. Ministério da Saúde. HumanizaSUS: gestão e formação nos processos de trabalho. Brasília: Ministério da Saúde, 2004.

BRASIL. Ministério da Ciência e Tecnologia. Conselho Nacional De Pesquisa. Diretório de Grupos de Pesquisa. Brasília, 2010. Disponível em: <http://dgp.cnpq.br/diretorioc/>. Acesso em: ago 2010a.

BRASIL. Ministério da Saúde. Sistema Pesquisa Saúde. Brasília, 2010. Disponível em: <http://portal2.saude.gov.br/sisct/>. Acesso em: ago 2010b.

BRASIL. Ministério da Educação e Cultura. Coordenação de Aperfeiçoamento de Pessoal de Nível Superior. Banco de Teses e Dissertaçôes. Brasília: MEC, 2010. Disponível em: <http://www.capes.gov.br/servicos/banco-de-teses>. Acesso em: ago 2010c.

GONCZI, A. Problemas asociados con la implementación de la educación basada en la competencia: de lo atomístico a lo holístico. In: CINTERFOR (Org.). Formación basada en competencia laboral. Situación actual y perspectivas, Montevideo: Cinterfor, 1997.p.161-169. HENNINGTON, E.L. Gestão dos processos de trabalho e humanização em saúde: reflexões a partir da ergologia. Revista de Saúde Pública. São Paulo, n.42, v.3, p.555-561, 2008.

LE DEIST, F.D.; WINTERTON, J. What is competence? Human Resource Development International, Tilburg, v.8, n.1, p.27-46, 2005.

LILLEY, K.C.; STEWART, D.E. The Australian preventive health agenda: what will this mean for workforce development? Australia \& New Zealand Health Policy. Collingwood, n.6, v.1, p.14, 2009.

MCMULLAN, M. Competence and its assessment: a review of the literature. British Journal of Pediatry. London, v.8, n.2, p.49-52, 2005.

NASCIMENTO, D.D.G.; OLIVEIRA, M.A.C. Reflexões sobre as competências profissionais para o processo de trabalho nos Núcleos de Apoio à Saúde da Família. $O$ Mundo da Saúde. São Paulo, n.34, v.1, p.92-96, 2010.

SANDBERG, J. Understanding human competence at work: an interpretative approach. Academy of Management Journal. New York, v.43, n.1, p.9-25, 2000.

SCIENTIFIC ELETRONIC LIBRARY ONLINE. SciELO. Disponível em: <http://www. scielo.org/php/index.php>. Acesso em: ago 2010.

SENAI. Rotas estratégicas para o futuro da indústria paranaense: roadmapping de saúde. Curitiba: SENAI/PR, 2008. 62 p.

\section{Nota}

${ }^{1}$ T.B.O. Fragelli trabalhou na concepção, pesquisa, análise e interpretação dos dados e redação do artigo. H.E. Shimizu trabalhou na formatação e revisão da versão a ser publicada. 


\section{Prospections for development of public policies for health professionals training from the analysis of the Brazilian scenario of competencies}

When referring to Public Health, literature points to the need for professionals who are able to work in different contexts. In this perspective, the theme of "competency" emerges from the administrative context for health in order to promote debates in the field of human resource development and training. This paper aims to present an overview of the Brazilian scene on professional skills in health for better understanding the construct at the national level and propose future research. The study used data from the Directory of Research Groups of the National Research Council (CNPq), the database of the Department of Science and Technology of the Ministry of Health (Health Research System), Bank of Theses and Dissertations of the Coordination for Higher Education Personnel (CAPES) and SciELO database. We observed inequality in the distribution of scientific researchers and little encouraged production. Thus greater efforts in the field of health management are necessary to build the theme.

> Key words: competencies; health professionals; public policies. 